\title{
Null-controllability of linear hyperbolic systems in one dimensional space
}

\author{
Jean-Michel Coron $^{\mathrm{a}, *, 1}$, Hoai-Minh Nguyen $^{\mathrm{b}, 1}$ \\ a Sorbonne Université, Université, Paris-Diderot SPC, CNRS, INRIA, Laboratoire Jacques-Louis Lions, équipe Cage, Paris, France \\ ${ }^{\mathrm{b}}$ Department of Mathematics, EPFL SB CAMA, Station 8 CH-1015 Lausanne, Switzerland
}

\section{A R T I C L E I N F O}

\section{Article history:}

Received 27 October 2019

Received in revised form 2 December 2020

Accepted 4 December 2020

Available online $\mathrm{xxxx}$

\section{Keywords:}

Null-controllability

Hyperbolic systems

Backstepping

Hilbert uniqueness method

Compactness

\begin{abstract}
A B S T R A C T
This paper is devoted to the controllability of a general linear hyperbolic system in one space dimension using boundary controls on one side. Under precise and generic assumptions on the boundary conditions on the other side, we previously established the optimal time for the null and the exact controllability for this system for a generic source term. In this work, we prove the null-controllability for any time greater than the optimal time and for any source term. Similar results for the exact controllability are also discussed.
\end{abstract}

CC 2020 Elsevier B.V. All rights reserved.

\section{Introduction and statement of the main result}

Linear hyperbolic systems in one dimensional space are frequently used in modeling of many systems such as traffic flow, heat exchangers, and fluids in open channels. The stability and boundary stabilization of these hyperbolic systems have been studied intensively in the literature, see, e.g., [1] and the references therein. In this paper, we are concerned about the optimal time for the null-controllability using boundary controls on one side. More precisely, we consider the system

$$
\begin{gathered}
\partial_{t} w(t, x)=\Sigma(x) \partial_{x} w(t, x)+C(x) w(t, x) \\
\text { for }(t, x) \in \mathbb{R}_{+} \times(0,1) .
\end{gathered}
$$

Here $w=\left(w_{1}, \ldots, w_{n}\right)^{\top}: \mathbb{R}_{+} \times(0,1) \rightarrow \mathbb{R}^{n}(n \geq 2), \Sigma$ and $C$ are $(n \times n)$ real matrix-valued functions defined in $[0,1]$. As usual, see e.g. [2], we assume that, may be after a linear change of variables $w \rightarrow R(x) w, \Sigma(x)$ is of the form

$$
\begin{aligned}
\Sigma(x)= & \operatorname{diag}\left(-\lambda_{1}(x), \ldots,-\lambda_{k}(x),\right. \\
& \left.\lambda_{k+1}(x), \ldots, \lambda_{n}(x)\right),
\end{aligned}
$$

where, with $n=m+k$,

$$
-\lambda_{1}(x)<\cdots<-\lambda_{k}(x)<0
$$

\footnotetext{
* Corresponding author.

E-mail addresses: coron@ann.jussieu.fr (J.-M. Coron), hoai-minh.nguyen@epfl.ch (H.-M. Nguyen).

1 The authors are partially supported by ANR, France Finite4SoS ANR-15CE23-0007.
}

$$
<\lambda_{k+1}(x)<\cdots<\lambda_{k+m}(x) .
$$

Throughout the paper, we assume that

$\lambda_{i}$ is Lipschitz on $[0,1]$ for $1 \leq i \leq n$

and

$C \in\left[L^{\infty}(0,1)\right]^{n \times n}$.

We are interested in the following type of boundary conditions and boundary controls. The boundary conditions at $x=0$ are given by

$w_{-}(t, 0)=B w_{+}(t, 0)$ for $t \geq 0$,

where $w_{-}=\left(w_{1}, \ldots, w_{k}\right)^{\top}$ and $w_{+}=\left(w_{k+1}, \ldots, w_{k+m}\right)^{\top}$, for some given $(k \times m)$ real, constant matrix $B$, and the boundary controls at $x=1$ are

$w_{+}(t, 1)=W(t)$ for $t \geq 0$,

where $W=\left(W_{k+1}, \ldots, W_{k+m}\right)^{\top}$ are controls.

Let us recall that the control system (1.1), (1.6), and (1.7) is null-controllable (resp. exactly controllable) at the time $T>0$ if, for every initial data $w_{0}:(0,1) \rightarrow \mathbb{R}^{n}$ in $\left[L^{2}(0,1)\right]^{n}$ (resp. for every initial data $w_{0}:(0,1) \rightarrow \mathbb{R}^{n}$ in $\left[L^{2}(0,1)\right]^{n}$ and for every (final) state $w_{T}:(0,1) \rightarrow \mathbb{R}^{n}$ in $\left.\left[L^{2}(0,1)\right]^{n}\right)$, there is a control $W:(0, T) \rightarrow \mathbb{R}^{m}$ in $\left[L^{2}(0, T)\right]^{m}$ such that the solution of (1.1), (1.6), and (1.7) satisfying $w(0, x)=w_{0}(x)$ vanishes (resp. reaches $\left.w_{T}\right)$ at the time $T: w(T, x)=0\left(\right.$ resp. $w(T, x)=w_{T}(x)$ ). Throughout this paper, we consider broad solutions in $L^{2}$ with respect to $t$ and $x$ for an initial data in $L^{2}(0,1)$ as in [2, Definition 
3.1]. In particular, the solutions belong to $C\left([0, T] ;\left[L^{2}(0,1)\right]^{n}\right)$ and $C\left([0,1] ;\left[L^{2}(0, T)\right]^{n}\right)$. The well-posedness for broad solutions was given in [2, Lemma 3.2]. In fact, in [2, Definition 3.1] and [2, Lemma 3.2], bounded broad solutions with respect to $t$ and $x$ for an initial data in $\left[L^{\infty}(0,1)\right]^{n}$ are considered, nevertheless, the extension for $L^{2}$-setting is quite straightforward (see also [3]). Set

$\tau_{i}:=\int_{0}^{1} \frac{1}{\lambda_{i}(\xi)} d \xi$ for $1 \leq i \leq n$

and

$T_{\text {opt }}:=\left\{\begin{array}{r}\max \left\{\tau_{1}+\tau_{m+1}, \ldots, \tau_{k}+\tau_{m+k},\right. \\ \left.\tau_{k+1}\right\} \text { if } m \geq k, \\ \max \left\{\tau_{k+1-m}+\tau_{k+1}, \tau_{k+2-m}+\tau_{k+2},\right. \\ \left.\ldots, \tau_{k}+\tau_{k+m}\right\} \text { if } m<k\end{array}\right.$

In this paper, we are mainly concerned about the optimal time for the null controllability of (1.1), (1.6), and (1.7) for $k \geq m \geq 1$. The null-controllability was known from [4, Section 5] for the time $\tau_{k}+\tau_{k+1}$ without any assumption on $B$ (see also [5-8] for feedback controls using backstepping). In our previous work [2], we established the null controllability of (1.1), (1.6), and (1.7) at the optimal time $T_{o p t}$ with $B \in \mathcal{B}$ defined in (1.10), for a generic $C$, i.e. for $\gamma C$ with $\gamma \in \mathbb{R}$ outside a discrete subset of $\gamma \in \mathbb{R}$. When $C \equiv 0$, we also show that there exists a linear time independent feedback which yields the null-controllability at the time $T_{o p t}$. Similar results for the exact controllability at $T_{o p t}$ were also established there (see Section 3 for a discussion). The optimality of $T_{\text {opt }}$ even for $C \equiv 0$ was also discussed in [2]. It is worth noting that there are choices of constants $\Sigma, B$, and $C$ when $m=2$ and $k \geq 2$ so that the system is not null-controllable at the time $T_{o p t}$ [2, part 2 of Theorem 1] (see also [4, pages 559561]). It is easy to see that $\mathcal{B}$ is an open subset of the set of (real) $k \times m$ matrices and the Hausdorff dimension of its complement is $\min \{k, m-1\}$.

In this work, we prove the null-controllability of (1.1), (1.6), and (1.7) for any time greater than $T_{\text {opt }}$ and for $m \geq k \geq 1$ without the generic requirement. Here is the main result of our paper:

Theorem 1. Let $k \geq m \geq 1$, and set

$\mathcal{B}:=\left\{B \in \mathbb{R}^{k \times m} ;\right.$ such that (1.11) holds

for $1 \leq i \leq \min \{k, m-1\}\}$,

where

the $i \times i$ matrix formed from the last $i$
columns and rows of $B$ is invertible.

Assume that $B \in \mathcal{B}$. The control system (1.1), (1.6), and (1.7) is null-controllable at any time $T>T_{\text {opt }}$.

To our knowledge, the null-controllability result of Theorem 1 in the case $m<k$ with general $m$ and $k$ is new. The sharpest known result on the time to obtain the null-controllability is $\tau_{k}+\tau_{k+1}$. When $m=k$, Theorem 1 can be derived from the exact controllable result in [9] under the additional assumption that (1.11) holds for $i=k$ (see Section 3 for a discussion). The starting point of our analysis is the backstepping approach. More precisely, as in [2] (see also [10] and the references therein for prior works), we make the following change of variables

$u(t, x)=w(t, x)-\int_{0}^{x} K(x, y) w(t, y) d y$.

Here the kernel $K: \mathcal{T}=\left\{(x, y) \in(0,1)^{2} ; 0<y<x\right\} \rightarrow \mathbb{R}^{n}$ is chosen such that $u$ satisfies

$\partial_{t} u(t, x)=\Sigma(x) \partial_{x} u(t, x)+S(x) u(t, 0)$

$$
\text { for }(t, x) \in(0, T) \times(0,1),
$$

where $S \in\left[L^{\infty}(0,1)\right]^{n \times n}$ has the structure

$S=\left(\begin{array}{cc}0_{k, k} & S_{-+} \\ 0_{m, k} & S_{++}\end{array}\right)$

with

$\left(S_{++}\right)_{p q}=0$ for $1 \leq q \leq p$,

$S_{-+} \in\left[L^{\infty}(0,1)\right]^{k \times m}$ and $S_{++} \in\left[L^{\infty}(0,1)\right]^{m \times m}$. Here and in what follows, $0_{i, j}$ denotes the zero matrix of size $i \times j$ for $i, j \in \mathbb{N}$, and $M_{p q}$ denotes the $(p, q)$-component of a matrix $M$. It is shown in [2, Proposition 3.5] that the null-controllability of (1.1), (1.6), and (1.7) at the time $T$ can be derived from the null-controllability at the time $T$ of (1.12) equipped the boundary condition at $x=0$

$u_{-}(t, 0)=B u_{+}(t, 0)$ for $t \geq 0$,

and the boundary controls at $x=1$

$u_{+}(t, 1)=U(t)$ for $t \geq 0$ where $U$ is the control.

To establish the null-controllability for $u$, we use the Hilbert uniqueness method which involves crucially a compactness type result in Lemma 4 with its roots in [2].

The backstepping approach for the control of partial differential equations was pioneered by Miroslav Krstic and his coauthors (see [11] for a concise introduction). The use of backstepping method to obtain the null-controllability for hyperbolic systems in one dimension was initiated in [7] for the case $m=k=$ 1 . This approach has been developed later on for more general $m$ and $k$ in $[5,6,8]$. In the case $n=2$ and for a special $\Sigma$, a similar change of variables was considered by Russell [4, Section 4] to obtain a canonical form. The backstepping method is now frequently used for various control problems modeling by partial differential equations in one dimension. For example, it has been also used to stabilize the wave equation [12-14], the parabolic equations in [15,16], nonlinear parabolic equations [17], and to obtain the null-controllability of the heat equation [18]. The standard backstepping approach relies on the Volterra transform of the second kind. It is worth noting that, in some situations, more general transformations have to be considered as for Korteweg-de Vries equations [19], Kuramoto-Sivashinsky equations [20], Schrödinger's equation [21], and hyperbolic equations with internal controls [22].

The rest of the paper is organized as follows: In Section 2, we establish Theorem 1. The exact controllability is discussed in Section 3.

\section{Optimal time for the null-controllability}

In this section, we study the null-controllability of (1.12) and (1.14) under the control law (1.15). The main result of this section, which implies Theorem 1 by [2, Proposition 3.5], is:

Theorem 2. Let $k \geq m \geq 1$ and assume that $B \in \mathcal{B}$. System (1.12) and (1.14) under the control law (1.15) is null-controllable at any time $T>T_{\text {opt }}$.

The rest of this section contains two sections. In the first section, we present some lemmas used in the proof of Theorem 2. The proof of Theorem 2 is given in the second section.

\subsection{Some useful lemmas}

Fix $T>0$. Define

$$
\begin{array}{ccc}
\mathcal{F}_{T}:\left[L^{2}(0, T)\right]^{m} & \rightarrow & {\left[L^{2}(0,1)\right]^{n}} \\
U & \mapsto & u(T, \cdot),
\end{array}
$$


where $u$ is the solution of the system (1.12)-(1.15) with $u(t=$ $0, \cdot)=0$.

In what follows, we denote

$\Sigma_{-}(x)=\operatorname{diag}\left(-\lambda_{1}(x), \ldots,-\lambda_{k}(x)\right)$,

$\Sigma_{+}(x)=\operatorname{diag}\left(\lambda_{k+1}(x), \ldots, \lambda_{k+m}(x)\right)$.

We begin with

Lemma 1. We have, for $V \in\left[L^{2}(0,1)\right]^{n}$,

$\mathcal{F}_{T}^{*}(V)=\Sigma_{+}(1) v_{+}(\cdot, 1)$ in $(0, T)$,

where $v$ is the unique solution of the system

$\begin{aligned} \partial_{t} v(t, x)= & \Sigma(x) \partial_{x} v(t, x)+\Sigma^{\prime}(x) v(t, x) \\ & \text { for }(t, x) \in(-\infty, T) \times(0,1),\end{aligned}$

with, for $t<T$,

$v_{-}(t, 1)=0$,

$$
\begin{aligned}
& \Sigma_{+}(0) v_{+}(t, 0)=-B^{\top} \Sigma_{-}(0) v_{-}(t, 0) \\
& \quad+\int_{0}^{1} S_{-+}^{\top}(x) v_{-}(t, x)+S_{++}^{\top}(x) v_{+}(t, x) d x,
\end{aligned}
$$

and

$v(t=T, \cdot)=V$ in $(0,1)$.

Throughout this paper, $\langle\cdot, \cdot\rangle$ denotes the Euclidean scalar product in the Euclidean space and $\langle\cdot, \cdot\rangle_{L^{2}(a, b)}$ denotes the scalar product in $\left[L^{2}(a, b)\right]^{\ell}$ for $a<b$ and $\ell \geq 1$.

Proof. We have

$$
\begin{aligned}
& \left\langle U, \mathcal{F}_{T}^{*} V\right\rangle_{L^{2}(0, T)} \\
= & \left\langle\mathcal{F}_{T} U, V\right\rangle_{L^{2}(0,1)}=\langle u(T, \cdot), v(T, \cdot)\rangle_{L^{2}(0,1)} \\
= & \int_{0}^{T} \partial_{t}\langle u(t, \cdot), v(t, \cdot)\rangle_{L^{2}(0,1)} d t \\
= & \int_{0}^{T}\left\langle\partial_{t} u(t, \cdot), v(t, \cdot)\right\rangle_{L^{2}(0,1)}+\left\langle u(t, \cdot), \partial_{t} v(t, \cdot)\right\rangle_{L^{2}(0,1)} d t \\
= & \int_{0}^{T} \int_{0}^{1}\left\langle\Sigma(x) \partial_{x} u(t, x)+S(x) u(t, 0), v(t, x)\right\rangle \\
& \quad+\left\langle u(t, x), \partial_{t} v(t, x)\right\rangle d x d t \text { by }(1.12) .
\end{aligned}
$$

An integration by parts yields

$\int_{0}^{T} \int_{0}^{1}\left\langle\Sigma(x) \partial_{x} u(t, x), v(t, x)\right\rangle d x d t$

$=\int_{0}^{T} \int_{0}^{1}-\left\langle\Sigma^{\prime}(x) v(t, x)+\Sigma(x) \partial_{x} v(t, x), u(t, x)\right\rangle d t$

$+\int_{0}^{T}\langle u(t, 1), \Sigma(1) v(t, 1)\rangle-\int_{0}^{T}\langle u(t, 0), \Sigma(0) v(t, 0)\rangle d t$.

Using the conditions on $u$ at $x=0$ and $x=1$ (see (1.14) and (1.15)), and (2.2), we have

$$
\begin{aligned}
& \int_{0}^{T}\langle u(t, 1), \Sigma(1) v(t, 1)\rangle-\int_{0}^{T}\langle u(t, 0), \Sigma(0) v(t, 0)\rangle d t \\
& =\int_{0}^{T}\left\langle\Sigma_{+} v_{+}, u_{+}\right\rangle(t, 1) d t \\
& \quad-\int_{0}^{T}\left\langle B^{\top} \Sigma_{-} v_{-}+\Sigma_{+} v_{+}, u_{+}\right\rangle(t, 0) d t .
\end{aligned}
$$

We then obtain

$$
\begin{aligned}
\left\langle U, \mathcal{F}_{T}^{*} V\right\rangle= & \int_{0}^{T} \int_{0}^{1}\langle S(x) u(t, 0), v(t, x)\rangle \\
& +\int_{0}^{T}\left\langle\Sigma_{+} v_{+}, u_{+}\right\rangle(t, 1) d t \\
& -\int_{0}^{T}\left\langle B^{T} \Sigma_{-} v_{-}+\Sigma_{+} v_{+}, u_{+}\right\rangle(t, 0) d t .
\end{aligned}
$$

Using the boundary condition (2.3), we obtain

$\left\langle U, \mathcal{F}_{T}^{*} V\right\rangle_{L^{2}(0, T)}=\int_{0}^{T}\left\langle\Sigma_{+} v_{+}, u_{+}\right\rangle(t, 1) d t$,

which implies the conclusion.

Similarly, we have the following result whose proof is omitted.

Lemma 2. Let $T>0$ and $u_{0} \in\left[L^{2}(0,1)\right]^{n}$. Assume that $u$ is the unique solution of (1.12) and (1.14) with $u(t=0, \cdot)=u_{0}$ and $u_{+}(\cdot, 0)=0$ for $t>0$. Then, for $V \in\left[L^{2}(0,1)\right]^{n}$, we have

$\int_{0}^{1}\langle u(T, x), V(x)\rangle d x=\int_{0}^{1}\left\langle u_{0}(x), v(0, x)\right\rangle d x$

where $v$ is the solution of (2.1)-(2.4).

Combining Lemmas 1 and 2, making a translation in time, and applying the Hilbert uniqueness method (see e.g. [23, Chapter 2]), we obtain

Lemma 3. Let $T>0$. System (1.12)-(1.15) is null controllable at the time $T$ if and only if, for some positive constant $C$,

$\int_{-T}^{0}\left|v_{+}(t, 1)\right|^{2} d t \geq C \int_{0}^{1}|v(-T, x)|^{2} d x \forall V \in\left[L^{2}(0,1)\right]^{n}$,

where $v$ is the unique solution of the system

$\begin{aligned} \partial_{t} v(t, x)= & \Sigma(x) \partial_{x} v(t, x)+\Sigma^{\prime}(x) v(t, x) \\ & \text { for }(t, x) \in(-\infty, 0) \times(0,1),\end{aligned}$

with, $t<0$,

$v_{-}(t, 1)=0$,

$$
\begin{aligned}
\Sigma_{+}(0) v_{+}(t, 0)= & -B^{\top} \Sigma_{-}(0) v_{-}(t, 0) \\
& +\int_{0}^{1} S_{-+}^{\top}(x) v_{-}(t, x)+S_{++}^{\top}(x) v_{+}(t, x) d x
\end{aligned}
$$

and

$v(t=0, \cdot)=V$ in $(0,1)$.

Finally, we establish a compactness type result which is one of the key ingredients in the proof of Theorem 2.

Lemma 4. Let $k \geq m \geq 1, B \in \mathcal{B}$, and $T \geq T_{\text {opt }}$. Assume that $\left(v_{N}\right)$ is a sequence of solutions of $(2.6)-(2.8)\left(\right.$ with $v_{N}(0, \cdot)$ in $\left[L^{2}(0,1)\right]^{n}$ ) such that

$$
\begin{aligned}
& \sup _{N}\left\|v_{N}(-T, \cdot)\right\|_{L^{2}(0,1)}<+\infty, \\
& \lim _{N \rightarrow+\infty}\left\|v_{N,+}(\cdot, 1)\right\|_{L^{2}(-T, 0)}=0 .
\end{aligned}
$$

We have, up to a subsequence,

$v_{N}(-T, \cdot)$ converges in $\left[L^{2}(0,1)\right]^{n}$,

and the limit $V \in\left[L^{2}(0,1)\right]^{n}$ satisfies the equation

$V=\mathcal{K} V$,

for some compact operator $\mathcal{K}$ from $\left[L^{2}(0,1)\right]^{n}$ into itself. Moreover, $\mathcal{K}$ depends only on $\Sigma, S$, and $B$; in particular, $\mathcal{K}$ is independent of $T$. 


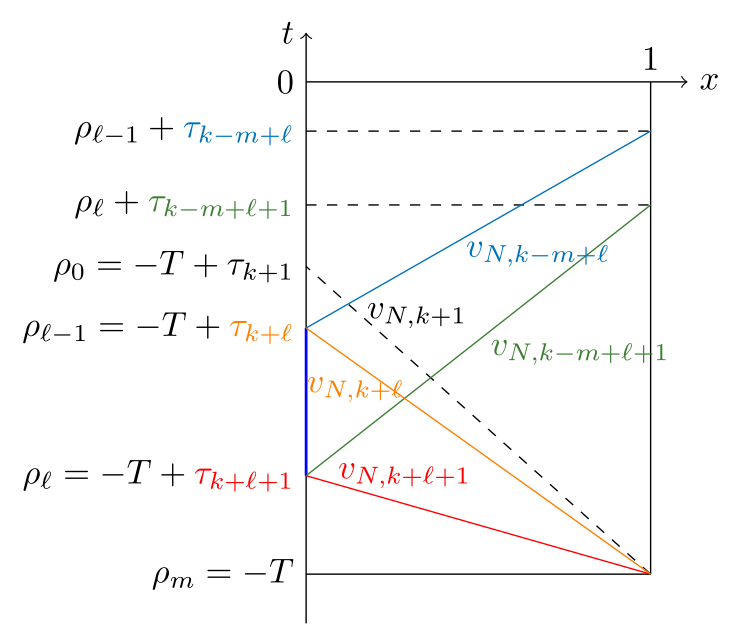

Fig. 1. The geometry associated to Lemma 4 when $\Sigma$ is constant and $1 \leq \ell \leq m$.

Proof. Set, for $0 \leq \ell \leq m-1$,

$\rho_{\ell}=-T+\tau_{k+\ell+1}$,

and denote $\rho_{m}=-T$. Use the characteristic method and the diagonal form of (2.6), and take into account (2.7) and (2.11). Since $\Sigma$ is Lipschitz, one can check that (see Fig. 1 ): for $1 \leq \ell \leq$ $m$,

$$
\begin{aligned}
& \text { (A1) for } 1 \leq j \leq k-m+\ell, \\
& \quad v_{N, j}=0 \text { in }\left(-T, \rho_{\ell-1}\right) \times(0,1),
\end{aligned}
$$

(A2) for $k+\ell+1 \leq j \leq k+m$,

$$
\lim _{N \rightarrow+\infty}\left\|v_{N, j}\right\|_{L^{2}\left(\rho_{\ell}, \rho_{\ell-1}\right)}=0
$$

(A3) for $t \in\left(\rho_{\ell}, \rho_{\ell-1}\right)$ and for $k+\ell+1 \leq j \leq k+m$,

$$
\lim _{N \rightarrow+\infty}\left\|v_{N, j}(t, \cdot)\right\|_{L^{2}(0,1)}=0 .
$$

We have, by (2.8), that for $t \in(-T, 0)$,

$$
\begin{aligned}
& \Sigma_{+}(0) v_{N,+}(t, 0)=-B^{\top} \Sigma_{-}(0) v_{N,-}(t, 0) \\
& +\int_{0}^{1} S_{-+}^{\top}(x) v_{N,-}(t, x)+S_{++}^{\top}(x) v_{N,+}(t, x) d x .
\end{aligned}
$$

Denote, for $1 \leq \ell \leq m$,

$V_{N, \ell}=\left(v_{N, k-m+\ell+1}, \ldots, v_{N, k}\right)^{\top}$,

$W_{N, \ell}=\left(v_{N, k+1}, \ldots, v_{N, k+\ell}\right)^{\top}$,

and set, for $1 \leq \ell \leq m$,

$\hat{\mathcal{D}}_{\ell}=\left\{(t, s): t \in\left(\rho_{\ell}, \rho_{\ell-1}\right), \rho_{m} \leq s \leq t\right\}$

and

$$
\mathcal{D}_{\ell}=\left\{(t, s): t \in\left(\rho_{\ell}, \rho_{\ell-1}\right), t \leq s \leq \rho_{0}\right\} .
$$

Let $1 \leq \ell \leq m-1$. Consider the last $(m-\ell)$ equations of (2.17) for $t \in\left(\rho_{\ell}, \rho_{\ell-1}\right)$. Multiply the system by the inverse of the matrix given in (1.11) with $i=m-\ell$. Using (A1), one can ignore the contribution of $v_{N, j}(t, 0)$ and $v_{N, j}(t, \cdot)$ for $1 \leq j \leq k-m+\ell$. View $v_{N, j}(\cdot, 0)$ and $v_{N, j}(t, \cdot)$ with $k+\ell+1 \leq j \leq k+m$ as perturbations. Applying the characteristic method and taking into account the diagonal form of (2.6), we obtain the following relation between
$V_{N, \ell}(\cdot, 0)$ and $W_{N, \ell}(\cdot, 0)$ : for $t \in\left(\rho_{\ell}, \rho_{\ell-1}\right)$,

$$
\begin{aligned}
V_{N, \ell}(t, 0)= & \int_{\rho_{m}}^{t} G_{\ell}(t, s) V_{N, \ell}(s, 0) d s \\
& +\int_{t}^{\rho_{0}} H_{\ell}(t, s) W_{N, \ell}(s) d s+F_{N, \ell}(t),
\end{aligned}
$$

for some $G_{\ell} \in\left[L^{\infty}\left(\hat{\mathcal{D}}_{\ell}\right)\right]^{(m-\ell) \times(m-\ell)}$ and $H_{\ell} \in$ $\left[L^{\infty}\left(\mathcal{D}_{\ell}\right)\right]^{(m-\ell) \times \ell}$ which depend only on $\Sigma, B$, and $S$, and for some $F_{N, \ell} \in\left[L^{2}\left(\rho_{\ell}, \rho_{\ell-1}\right)\right]^{m-\ell}$ which depends only on $\Sigma, B$, and $S$, and $v_{N, j}(\cdot, 0)$ and $v_{N, j}(t, \cdot)$ for $t \in\left(\rho_{\ell}, \rho_{\ell-1}\right)$ with $k+\ell+1 \leq j \leq k+m$. Moreover, by (A2) and (A3), one obtains

$F_{N, \ell} \rightarrow 0$ in $\left[L^{2}\left(\rho_{\ell}, \rho_{\ell-1}\right)\right]^{m-\ell}$ as $N \rightarrow+\infty$.

Let $1 \leq \ell \leq m$. Consider the first $\ell$ equations of (2.17) for $t \in\left(\rho_{\ell}, \rho_{\ell-1}\right)$. Since $\left(S_{++}^{\top}\right)_{p q}=0$ for $p \leq q \leq m$ by (1.13), $v_{N, k+j}$ with $j \geq l$ does not appear in the integral terms of the first $\ell$ equations of (2.17). Using (A1), one can ignore the contribution of $v_{N, j}(t, 0)$ and $v_{N, j}(t, \cdot)$ for $1 \leq j \leq k-m+\ell$. Applying the characteristic method and taking into account the diagonal form of (2.6), we have

$$
\begin{aligned}
W_{N, \ell}(t, 0)= & Q_{\ell} V_{N, \ell}(t, 0)+\int_{\rho_{m}}^{t} L_{\ell}(t, s) V_{N, \ell}(s, 0) d s \\
& +\int_{t}^{\rho_{0}} M_{\ell}(t, s) W_{N, \ell-1}(s, 0) d s .
\end{aligned}
$$

for some constant $Q_{\ell} \in \mathbb{R}^{\ell \times(m-\ell)}$, for some $L_{\ell} \in\left[L^{\infty}\left(\hat{\mathcal{D}}_{\ell}\right)\right]^{\ell \times(m-\ell)}$ and $M_{\ell} \in\left[L^{\infty}\left(\mathcal{D}_{\ell}\right)\right]^{\ell \times(\ell-1)}$, all depending only on $\Sigma, B$, and $S$. In the case $\ell=m,(2.19)$ is understood in the sense that the first two terms on the RHS are 0.

We are now in the position to derive the conclusion. We have, by the characteristic method and the diagonal form of (2.6),

(i) $v_{N,-}(-T, \cdot)=0$ in $(0,1)$;

(ii) the information of $v_{N,+}(-T, \cdot)$ in $(0,1)$ is encoded by the information of $v_{N, k+1}(\cdot, 0)$ on $\left(\rho_{m}, \rho_{0}\right)$, of $v_{N, k+2}(\cdot, 0)$ on $\left(\rho_{m}, \rho_{1}\right), \ldots$, of $v_{N, k+m}(\cdot, 0)$ on $\left(\rho_{m}, \rho_{m-1}\right)$;

(iii) Using (2.18) for $\ell=m-1$, one can solve $V_{N, m-1}(t, 0)$ for $t \in\left(\rho_{m-1}, \rho_{m-2}\right)$ as a function of $W_{N, m-1}(\cdot, 0)$ and $F_{N, m-1}(\cdot)$ in $\left(\rho_{m-1}, \rho_{m-2}\right)$, and $V_{N, m-1}(\cdot, 0)$ in $\left(\rho_{m}, \rho_{m-1}\right)$. Continue the process with $\ell=m-2$, then with $\ell=m-3, \ldots$, and finally with $\ell=1$. Note that, by (A1),

$$
v_{N, k-m+\ell+1}(\cdot, 0)=0 \text { in }\left(\rho_{m}, \rho_{\ell}\right) \text {. for } 0 \leq \ell \leq m-1 \text {. }
$$

One thus can solve

$V_{N, 1}(\cdot, 0) \in L^{2}\left(\rho_{2}, \rho_{0}\right) \times \cdots \times L^{2}\left(\rho_{m}, \rho_{0}\right)$

as a function of $W_{N, m-1}(\cdot, 0)$

$\in L^{2}\left(\rho_{m}, \rho_{0}\right) \times \cdots \times L^{2}\left(\rho_{m}, \rho_{m-2}\right)$ and $F_{N, j}(\cdot)$ with $j=$ $1, \ldots, m$, and one has

$V_{N, 1}(\cdot, 0)=\mathcal{K}_{1} W_{N, m-1}(\cdot, 0)+g_{N}$.

where $g_{N} \in L^{2}\left(\rho_{2}, \rho_{0}\right) \times \cdots \times L^{2}\left(\rho_{m}, \rho_{0}\right)$ converges to 0 in the corresponding $L^{2}$-norm and $\mathcal{K}_{1}$ is a compact operator depending only on $\Sigma, S$ and $B$.

The conclusion now follows from (2.19) applied to $1 \leq \ell \leq m$ noting that the first two terms on the RHS of (2.19) are 0 if $\ell=m$. The proof is complete.

Remark 1. The assumption $\left(S_{++}^{\top}\right)_{p q}=0$ for $p \leq q \leq m$ is essentially not necessary for the proof of Lemma 6 . Without this condition, there is an error term in (2.19) which goes to 0 in $\left[L^{2}\left(\rho_{\ell}, \rho_{\ell-1}\right)\right]^{\ell}$ as $N \rightarrow+\infty$ thanks to (A2) and (A3). The conclusion then follows similarly. 


\subsection{Proof of Theorem 2}

The arguments are in the spirit of $[24,25]$ (see also [26,27]). For $T>T_{\text {opt }}$, set

$Y_{T}:=\left\{V \in L^{2}(0,1): V\right.$ is the limit in $L^{2}(0,1)$ of some subsequence of solutions $\left(v_{N}(-T, \cdot)\right)$ of (2.6)-(2.8) such that (2.10) and (2.11) hold \} .

It is clear that $Y_{T}$ is a vectorial space. Moreover, by (2.13) and the compact property of $\mathcal{K}$, we have

$\operatorname{dim} Y_{T} \leq C$,

for some positive constant $C$ independent of $T$.

We next show that

$Y_{T_{2}} \subset Y_{T_{1}}$ for $T_{\text {opt }}<T_{1}<T_{2}$.

Indeed, let $V \in Y_{T_{2}}$. There exists a sequence of solutions $\left(v_{N}\right)$ of (2.6)-(2.8) such that

$\left\{\begin{array}{c}v_{N}\left(-T_{2}, \cdot\right) \rightarrow V \text { in } L^{2}(0,1), \\ \lim _{N \rightarrow+\infty}\left\|v_{N,+}(\cdot, 1)\right\|_{L^{2}\left(-T_{2}, 0\right)}=0 .\end{array}\right.$

By considering the sequence $v_{N}(\cdot-\tau, \cdot)$ with $\tau=T_{2}-T_{1}$, we derive that $V \in Y_{T_{1}}$.

By Lemma 3, to obtain the null-controllability at the time $T>T_{\text {opt }}$, it suffices to prove (2.5) by contradiction. Assume that there exists a sequence of solutions $\left(v_{N}\right)$ of $(2.6)-(2.8)$ such that

$N \int_{-T}^{0}\left|v_{N,+}(t, 1)\right|^{2} d t \leq \int_{0}^{1}\left|v_{N}(-T, x)\right|^{2} d x=1$.

By (2.12), up to a subsequence, $v_{N}(-T, \cdot)$ converges in $L^{2}(0,1)$ to a limit $V$. It is clear that $\|V\|_{L^{2}(0,1)}=1$; in particular, $V \neq 0$. Consequently,

$Y_{T} \neq\{0\}$.

By (2.21), (2.22), and (2.25), there exist $T_{\text {opt }}<T_{1}<T_{2}<T$ such that

$\operatorname{dim} Y_{T_{1}}=\operatorname{dim} Y_{T_{2}} \neq 0$.

We claim that, for $V \in Y_{T_{1}}$,

$\Sigma \partial_{x} V+\Sigma^{\prime} V$ is an element in $Y_{T_{1}}$.

Indeed, since $Y_{T_{1}}=Y_{T_{2}}$, by the definition of $Y_{T_{2}}$, there exists a sequence of solutions $\left(v_{N}\right)$ of $(2.6)-(2.8)$ such that

$\left\{\begin{array}{c}\lim _{N \rightarrow+\infty}\left\|v_{N,+}(\cdot, 1)\right\|_{L^{2}\left(-T_{2}, 0\right)}=0, \\ V=\lim _{N \rightarrow+\infty} v_{N}\left(-T_{2}, \cdot\right) \text { in } L^{2}(0,1) .\end{array}\right.$

Using (2.22), one may assume that $T_{2}-T_{1}$ is small. We claim that, for $t \in\left(-T_{2},-T_{1}\right]$,

$\sup _{N}\left\|v_{N}(t, \cdot)\right\|_{L^{2}(0,1)}<+\infty$.

Noting that $\Sigma$ and $\Sigma^{\prime}$ are diagonal, we have, by the characteristic method, for $t \in\left(-T_{2},-T_{\text {opt }}\right)$

$v_{N,-}(t, \cdot)=0$ in $(0,1)$.

Using the characteristic method again, we also have, for $t \in$ $\left(-T_{2},-T_{1}\right]$,

$$
\begin{aligned}
\left\|v_{N,+}(t, \cdot)\right\|_{L^{2}(0,1)} \leq & C\left(\left\|v_{N,+}\left(-T_{2}, \cdot\right)\right\|_{L^{2}(0,1)}\right. \\
& \left.+\left\|v_{N,+}(\cdot, 1)\right\|_{L^{2}\left(-T_{2}, t\right)}\right) .
\end{aligned}
$$

We derive from (2.27) that

$\sup _{N}\left\|v_{N,+}(t, \cdot)\right\|_{L^{2}(0,1)}<+\infty$.

Combining (2.29) and (2.31) yields (2.28).

Using (2.12), without loss of generality, one may assume that $v_{N}\left(-T_{1}, \cdot\right) \rightarrow \hat{V}$ in $L^{2}(0,1)$ for some $\hat{V} \in L^{2}(0,1)$.

Let $\hat{v}$ be the unique solution of the system

$$
\begin{aligned}
\partial_{t} \hat{v}(t, x)= & \Sigma(x) \partial_{x} \hat{v}(t, x)+\Sigma^{\prime}(x) \hat{v}(t, x) \\
& \text { for }(t, x) \in\left(-\infty,-T_{1}\right) \times(0,1),
\end{aligned}
$$

with, for $t<-T_{1}$,

$\hat{v}_{-}(t, 1)=0$,

$$
\begin{aligned}
& \Sigma_{+}(0) \hat{v}_{+}(t, 0)=-B^{\top} \Sigma_{-}(0) \hat{v}_{-}(t, 0) \\
& \quad+\int_{0}^{1} S_{-+}^{\top}(x) \hat{v}_{-}(t, x)+S_{++}^{\top}(x) \hat{v}_{+}(t, x) d x
\end{aligned}
$$

and

$\hat{v}\left(t=-T_{1}, \cdot\right)=\hat{V}$.

One then has, for $\tau<-T_{1}$,

$v_{N} \rightarrow \hat{v}$ in $C^{0}\left(\left[\tau,-T_{1}\right] ; L^{2}(0,1)\right)$.

In particular, by (2.22), we have

$\hat{v}(t, \cdot) \in Y_{T_{1}}$ for $t \in\left[-T_{2},-T_{1}\right)$

and

$V=\hat{v}\left(-T_{2}, \cdot\right)$ in $(0,1)$.

Since, in the distributional sense and hence in $Y_{T_{1}}$,

$\partial_{t} \hat{v}\left(-T_{2}, \cdot\right)=\lim _{\varepsilon \rightarrow 0_{+}} \frac{1}{\varepsilon}\left[\hat{v}\left(-T_{2}+\varepsilon, \cdot\right)-\hat{v}\left(-T_{2}, \cdot\right)\right]$,

and, for $\varepsilon>0$ small,

$\frac{1}{\varepsilon}\left[\hat{v}\left(-T_{2}+\varepsilon, \cdot\right)-\hat{v}\left(-T_{2}, \cdot\right)\right] \in Y_{T_{1}}$ by $(2.37)$,

one derives that

$\Sigma \partial_{x} \hat{v}\left(-T_{2}, \cdot\right)+\Sigma^{\prime} \hat{v}\left(-T_{2}, \cdot\right) \in Y_{T_{1}}$,

which implies (2.26). In particular, $Y_{T_{1}} \subset H^{1}(0,1)$.

Recall that $Y_{T_{1}}$ is real and of finite dimension. Consider its natural extension as a complex vectorial space and still denote its extension by $Y_{T_{1}}$. Define

$$
\begin{array}{ccc}
\mathcal{A}: \quad Y_{T_{1}} & \rightarrow & Y_{T_{1}} \\
V & \mapsto & \Sigma \partial_{\chi} V+\Sigma^{\prime} V .
\end{array}
$$

From the definition of $Y_{T_{1}}$, it is clear that, for $V \in Y_{T_{1}}$,

$V_{-}(1)=0$

and

$$
\begin{aligned}
& \Sigma_{+}(0) V_{+}(0)=-B^{\top} \Sigma_{-}(0) V_{-}(0) \\
& \quad+\int_{0}^{1} S_{-+}^{\top}(x) V_{-}(x)+S_{++}^{\top}(x) V_{+}(x) d x .
\end{aligned}
$$

Since $Y_{T_{1}} \neq\{0\}$ and $Y_{T_{1}}$ is of finite dimension, there exists $\lambda \in \mathbb{C}$ and $V \in Y_{T_{1}} \backslash\{0\}$ such that

$\mathcal{A} V=\lambda V$.

Set

$v(t, x)=e^{\lambda t} V(x)$ in $(-\infty, 0) \times(0,1)$. 
Using (2.38) and (2.39), one can verify that $v(t, x)$ satisfies (2.6)(2.8). Using (2.38) and applying the characteristic method, one deduces that

$v_{-}(t, \cdot)=0$ in $(0,1)$ for $t<-\tau_{k}$.

From (2.8), we then obtain, for $t<-\tau_{k}$,

$\Sigma_{+}(0) v_{+}(t, 0)=\int_{0}^{1} S_{++}^{\top}(x) v_{+}(t, x) d x$.

Using the structure of $S_{++}$, we then have

$v_{k+1}(t, 0)=0$ for $t<-\tau_{k}$.

By the characteristic method, this in turn implies that, for $t<$ $-\tau_{k}-\tau_{k+1}$.

$v_{k+1}(t, \cdot)=0$ in $(0,1)$.

Similarly, we have, for $t<-\tau_{k}-\tau_{k+1}-\tau_{k+2}$,

$v_{k+2}(t, \cdot)=0$ in $(0,1)$

$\ldots$, and for $t<-\tau_{k}-\tau_{k+1}-\cdots-\tau_{k+m}$,

$v_{k+m}(t, \cdot)=0$ in $(0,1)$.

Then $v(t, \cdot)=0$ in $(0,1)$ for $t<-\tau_{k}-\tau_{k+1}-\cdots-\tau_{k+m}$. It follows that $V=0$ which contradicts the fact $V \neq 0$. Thus (2.5) holds and the null-controllability is valid for $T>T_{\text {opt }}$.

\section{Optimal time for the exact controllability}

This section is on the exact controllability of (1.1), (1.6), and (1.7) for $m \geq k \geq 1$. We give two new proofs, one in the spirit of the proof of Theorem 1 and one is derived from Theorem 1, of the following result due to $\mathrm{Hu}$ and Olive [9].

Theorem 3. Assume that $m \geq k \geq 1$. Set

$\mathcal{B}_{e}:=\left\{B \in \mathbb{R}^{k \times m} ;\right.$ such that (1.11) holds for $\left.1 \leq i \leq k\right\}$,

Assume that $B \in \mathcal{B}_{e}$. The control system (1.1), (1.6), and (1.7) is exactly controllable at any time $T>T_{\text {opt }}$.

The exact controllability of (1.1), (1.6), and (1.7) for $m \geq k$ has been investigated intensively in the literature. When $m=k$ under a similar condition, this exact controllability was considered in [4, Theorem 3.2]. In the linear case with $m \geq k$ and $C \equiv$ 0 , the exact controllability was obtained by Weck [28]. In the quasilinear case with $m \geq k$, the exact controllability was derived in [29, Theorem 3.2] (see also [30]) for $m \geq k$ and for the time $\tau_{k}+\tau_{k+1}$ under a condition which is equivalent to the fact that (1.11) holds for $1 \leq i \leq k$. The result was improved when $C=0$ in [10] when the time of control is $\max \left\{\tau_{k+1}, \tau_{k}+\tau_{m+1}\right\}$ involving backstepping. The exact controllability of (1.1), (1.6), and (1.7) at the time $T_{o p t}$ was recently established in [2] for a generic $C$, i.e., for $\gamma C$ with $\gamma \in \mathbb{R}$ outside a discrete subset of $\gamma \in \mathbb{R}$ using the backstepping approach. The generic condition of $C$ is not required for $C$ with small $L^{\infty}$-norm by the same approach. It is worth noting that $\mathcal{B}_{e}$ is an open subset of the set of (real) $k \times m$ matrices and the Hausdorff dimension of its complement is $k$. The generic condition is then removed recently in [9] by a different approach. The optimal time for $B \notin \mathcal{B}_{e}$ was discussed in [2, Proposition 1.6] (see also [9]).

In this section, we first show how to adapt the approach for Theorem 1 to derive Theorem 3. As in the study of the null-controllability, it suffices, by [2, Proposition 3.1], to establish

Theorem 4. Let $m \geq k \geq 1$ and assume that $B \in \mathcal{B}_{e}$. System (1.12)-(1.14) under the control law (1.15) is exactly controllable at any time $T>T_{\text {opt }}$.

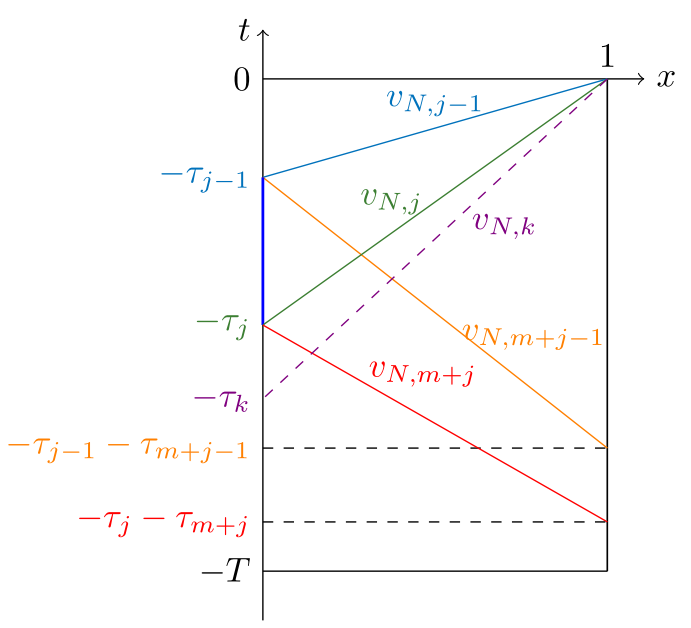

Fig. 2. The geometry associated to Lemma 6 when $\Sigma$ is constant and $1 \leq j \leq k$.

As a consequence of Lemma 1 , by the Hilbert uniqueness principle, see, e.g., [23, Chapter 2], we have

Lemma 5. Let $T>0$. System (1.12)-(1.15) is exactly controllable at the time $T$ if and only if, for some positive constant $C$,

$\int_{-T}^{0}\left|v_{+}(t, 1)\right|^{2} d t \geq C \int_{0}^{1}|v(0, x)|^{2} d x \forall V \in\left[L^{2}(0,1)\right]^{n}$,

for all solution $v$ of (2.6)-(2.8).

As a variant of Lemma 4, we establish

Lemma 6. Let $m \geq k \geq 1, B \in \mathcal{B}_{e}$, and $T \geq T_{\text {opt }}$. Assume that $\left(v_{N}\right)$ be a sequence of solutions of (2.6)-(2.8) such that

$\left\{\begin{array}{c}\sup _{N}\left\|v_{N}(0, \cdot)\right\|_{L^{2}(0,1)}<+\infty, \\ \lim _{N \rightarrow+\infty}\left\|v_{N,+}(\cdot, 1)\right\|_{L^{2}(0, T)}=0 .\end{array}\right.$

We have, up to a subsequence,

$v_{N}(0, \cdot)$ converges in $L^{2}(0,1)$,

and the limit $V \in\left[L^{2}(0,1)\right]^{n}$ satisfies the equation

$V=\mathcal{K}_{e} V$,

for some compact operator $\mathcal{K}_{e}$ from $\left[L^{2}(0,1)\right]^{n}$ into itself. Moreover, $\mathcal{K}_{e}$ depends only on $\Sigma, S$, and $B$; in particular, $\mathcal{K}_{e}$ is independent of $T$.

Proof. Denote $\tau_{0}=0$. Use the characteristic and diagonal form of (2.6), and take into account (2.7) and the limit in (3.2). Since $\Sigma$ is Lipschitz, one can check that, for $1 \leq j \leq k$ (see Fig. 2):

(B1) for $1 \leq \ell \leq j-1$,

$$
v_{N, \ell}=0 \text { in }\left(-T,-\tau_{j-1}\right) \times(0,1)
$$

(B2) for $m+j \leq \ell \leq m+k$,

$$
\lim _{N \rightarrow+\infty}\left\|v_{N, \ell}\right\|_{L^{2}\left(-\tau_{j},-\tau_{j-1}\right)}=0,
$$

(B3) for $t \in\left(-\tau_{j},-\tau_{j-1}\right)$ and for $m+j \leq \ell \leq m+k$,

$$
\lim _{N \rightarrow+\infty}\left\|v_{N, \ell}(t, \cdot)\right\|_{L^{2}(0,1)}=0
$$

We have, by (2.8), for $-T<t<0$,

$\Sigma_{+}(0) v_{N,+}(t, 0)=-B^{\top} \Sigma_{-}(0) v_{N,-}(t, 0)$

$+\int_{0}^{1} S_{-+}^{\top}(x) v_{N,-}(t, x)+S_{++}^{\top}(x) v_{N,+}(t, x) d x$. 
Denote, for $1 \leq j \leq k$,

$V_{N, j}^{e}=\left(v_{N, j}, \ldots, v_{N, k}\right)^{\top}$,

$W_{N, j}^{e}=\left(v_{N, k+1}, \ldots, v_{N, m+j-1}\right)^{\top}$,

and set, for $1 \leq j \leq k$,

$\hat{\mathcal{D}}_{j}^{e}:=\left\{(t, s): t \in\left(-\tau_{j},-\tau_{j-1}\right), t \leq s \leq 0\right\}$,

and

$\mathcal{D}_{j}^{e}:=\left\{(t, s): t \in\left(-\tau_{j},-\tau_{j-1}\right),-\tau_{k} \leq s \leq t\right\}$.

When $m=k$ and $j=1, W_{N, 1}^{e}$ is irrelevant.

Let $1 \leq j \leq k$. Consider the last $(k-j+1)$ equations of (3.8) for $t \in\left(-\tau_{j},-\tau_{j-1}\right)$. Multiply the system by the inverse of the matrix given in (1.11) with $i=k-j+1$. Using (B1), one can ignore the contribution of $v_{N, \ell}$ for $1 \leq \ell \leq j-1$. View $v_{N, \ell}(t, \cdot)$ for $x \in(0,1)$ and for $t \in\left(-\tau_{j},-\tau_{j-1}\right)$, and $v_{N, \ell}(\cdot, 0)$ for $t \in\left(-\tau_{j},-\tau_{j-1}\right)$ with $m+j \leq \ell \leq k+m$ as perturbations. Using the characteristic and the diagonal form of (2.6), we obtain the following relation between $V_{N, j}^{e}(\cdot, 0)$ and $W_{N, j}^{e}(\cdot, 0)$ : for $t \in\left(-\tau_{j},-\tau_{j-1}\right)$,

$$
\begin{aligned}
& V_{N, j}^{e}(t, 0)=\int_{-\tau_{k}}^{t} G_{j}^{e}(t, s) V_{N, j}^{e}(s, 0) d s \\
& +\int_{t}^{0} H_{j}^{e}(t, s) W_{N, j}^{e}(s, 0) d s+F_{N, j}^{e}(t),
\end{aligned}
$$

for some $G_{j}^{e} \in\left[L^{\infty}\left(\mathcal{D}_{j}^{e}\right)\right]^{(k-j+1) \times(k-j+1)}$ and $H_{j}^{e} \in\left[L^{\infty}\left(\hat{\mathcal{D}}_{j}^{e}\right)\right]^{(k-j+1) \times(m-k+j-1)}$ and which depends only on $\Sigma, S$, and $B$, and for some $F_{N, j}^{e} \in\left[L^{2}\left(-\tau_{j},-\tau_{j-1}\right)\right]^{k-j+1}$, which depends only on $\Sigma, S$, and $B$, and $v_{N,+}$. Moreover, by (B2) and (B3),

$F_{N, j}^{e} \rightarrow 0$ in $L^{2}\left(-\tau_{j},-\tau_{j-1}\right)$ as $N \rightarrow+\infty$.

When $k=m$ and $j=1$, the second term on the RHS of (3.9) is understood by 0 .

Let $1 \leq j \leq k$. Consider the first ( $m-k+j-1$ ) equations of (3.8) for $t \in\left(-\tau_{j},-\tau_{j-1}\right)$. Since $\left(S_{++}^{\top}\right)_{p q}=0$ for $p \leq q \leq m$ by (1.13), $v_{N, k+\ell}$ with $\ell \geq m-k+j-1$ does not appear in the integral terms of the first $(m-k+j-1)$ equations of (2.17), which is equivalent to the fact that $v_{N, \ell}$ with $\ell \geq m+j-1$ does not appear in the integral term of the first $(m-k+j-1)$ equations of (2.17). We have, by (B1), for $t \in\left(-\tau_{j},-\tau_{j-1}\right)$,

$$
\begin{aligned}
W_{N, j}^{e}(t, 0)= & Q_{j}^{e} V_{N, j}^{e}(t, 0)+\int_{-\tau_{k}}^{t} L_{j}^{e}(t, s) V_{N, j}^{e}(s, 0) d s \\
& +\int_{t}^{0} M_{\ell}^{e}(t, s) W_{N, j-1}^{e}(s, 0) d s .
\end{aligned}
$$

for some constant $Q_{j}^{e} \in \mathbb{R}^{(m-k+j-1) \times(k-j+1)}$, for some $L_{j}^{e} \in\left[L^{\infty}\left(\mathcal{D}_{j}^{e}\right)\right]^{(m-k+j-1) \times(k-j+1)}$, and for some $M_{j}^{e} \in$

$\left[L^{\infty}\left(\hat{\mathcal{D}}_{j}\right)\right]^{(m-k+j-1) \times(m-k+j-2)}$, all depending only on $\Sigma, B$, and $S$. When $k=m$ and $j=1$, (3.11) is irrelevant.

We are ready to derive the conclusion. Using (3.11) with $j=$ 1 , one can solve $W_{N, 1}^{e}(t, 0)$ as a function of $V_{N, 1}^{e}(t, 0)$ for $t \in$ $\left(-\tau_{1},-\tau_{0}\right)=\left(-\tau_{1}, 0\right)$ (if $m=k$, then this is irrelevant). Continue the process with $j=2$, then $j=3, \ldots$, finally with $j=k$. We thus obtain

$$
\begin{aligned}
W_{N, k}^{e} \in & {\left[L^{2}\left(-\tau_{k},-\tau_{0}\right)\right]^{m-k} \times L^{2}\left(-\tau_{k},-\tau_{1}\right) \times } \\
& \ldots \times L^{2}\left(-\tau_{k},-\tau_{k-1}\right)
\end{aligned}
$$

as a linear continuous function of $V_{N, k}^{e} \in L^{2}\left(-\tau_{1}, 0\right) \times \cdots \times$ $L^{2}\left(-\tau_{k}, 0\right)$. The conclusion now follows from (3.9) after noting that, by the characteristic method and the diagonal form of (2.6),

- $\lim _{N \rightarrow 0} v_{N,+}(0, \cdot)=0$ in $\left[L^{2}(0,1)\right]^{m}$.
- the information of $v_{N,-}(0, \cdot)$ is encoded by the information of $v_{N, 1}(\cdot, 0)$ on $\left(-\tau_{1}, 0\right)$, of $v_{N, 2}(\cdot, 0)$ on $\left(-\tau_{2}, 0\right), \ldots$, of $v_{N, k}(\cdot, 0)$ on $\left(-\tau_{k}, 0\right)$.

The proof is complete.

Remark 2. The assumption $\left(S_{++}^{\top}\right)_{p q}=0$ for $p \leq q \leq m$ is essentially not necessary for the proof of Lemma 6 . Without this condition, there is an error term in (3.11) which goes to 0 in $\left[L^{2}\left(-\tau_{j},-\tau_{j-1}\right)\right]^{m-k+j-1}$ as $N \rightarrow+\infty$ thanks to (B2) and (B3). The conclusion then follows similarly.

We are ready to give the

Proof of Theorem 4. The proof of Theorem 4 is similar to the one of Theorem 3. For $T>T_{\text {opt }}$, set

$Y_{T}^{e}:=\left\{V \in L^{2}(0,1): V\right.$ is the limit in $L^{2}(0,1)$ of some subsequence of solutions $\left(v_{N}(0, \cdot)\right)$ of (2.6)-(2.8) such that (3.2) holds $\}$.

As in Theorem 2, $Y_{T}^{e}$ is a vectorial space of finite dimension and there exist $T_{\text {opt }}<T_{1}<T_{2}<T$ such that

$\operatorname{dim} Y_{T_{1}}^{e}=\operatorname{dim} Y_{T_{2}}^{e}$.

Fix such $T_{1}$ and $T_{2}$. By Lemma 5 , it suffices to prove (3.1) by contradiction. Assume that (3.1) does not hold. Then, as in the proof Theorem 2, there exist $\lambda \in \mathbb{C}$ and $V \in Y_{T_{1}}^{e} \backslash\{0\}$ such that

$\Sigma \partial_{x} V+\Sigma^{\prime} V=\lambda V$.

Set

$v(t, x)=e^{\lambda t} V(x)$ in $(-\infty, 0) \times(0,1)$.

As in the proof of Theorem 2 , one can verify that $v(\cdot, \cdot)$ satisfies (2.6)-(2.8). Applying the characteristic method, one deduces that

$v_{-}(t, \cdot)=0$ for $t<-\tau_{k}$.

As in the proof of Theorem 2, we also have

$v(t, \cdot)=0$ in $(0,1)$ for $t<-\tau_{k}-\tau_{k+1}-\cdots-\tau_{k+m}$.

It follows that $V=0$ which contradicts the fact $V \neq 0$. Thus (3.1) holds and the exact-controllability is valid for $T>T_{\text {opt }}$.

We next give

The second proof of Theorem 3. The second proof of Theorem 3 can be also deduced from Theorem 1 . Indeed, consider first the case $m=k$. By making a change of variables

$\widetilde{w}(t, x)=w(T-t, x)$ for $t \in(0, T), x \in(0,1)$.

Then

$\widetilde{w}_{-}(t, 0)=\widetilde{B}^{-1} \widetilde{w}_{+}(t, 0)$,

with $\widetilde{w}_{-}(t, \cdot)=\left(w_{2 k}, \ldots \dot{\sim}, w_{k+1}\right)^{\top}(T-t, \cdot)$, and $\widetilde{w}_{+}(t, \cdot)=$ $\left(w_{k}, \ldots, w_{1}\right)^{\top}(T-t, \cdot)$, and $\widetilde{B}_{i j}=B_{p q}$ with $p=k-i$ and $q=k-j$. Note that the $i \times i$ matrix formed from the first $i$ columns and rows of $\widetilde{B}$ is invertible. Using Gaussian elimination method, one can find $(k \times k)$ matrices $T_{1}, \ldots, T_{N}$ such that

$T_{N} \ldots T_{1} \widetilde{B}=U$

where $U$ is a $(k \times k)$ upper triangular matrix, and $T_{i}(1 \leq i \leq N)$ is the matrix given by the operation which replaces a row $p$ by itself plus a multiple of a row $q$ for some $1 \leq q<p \leq N$. It follows that

$\widetilde{B}^{-1}=U^{-1} T_{N} \ldots T_{1}$. 
One can check that $U^{-1}$ is an invertible, upper triangular matrix and $T_{N} \ldots T_{1}$ is an invertible, lower triangular matrix. It follows that the $i \times i$ matrix formed from the last $i$ columns and rows of $\widetilde{B}^{-1}$ is the product of the matrix formed from the last $i$ columns and rows of $U^{-1}$ and the matrix formed from the last $i$ columns and rows of $T_{N} \ldots T_{1}$. Therefore, $\widetilde{B}^{-1} \in \mathcal{B}$. One can also check that the exact controllability of the system for $w(\cdot, \cdot)$ at the time $T$ is equivalent to the null-controllability of the system for $\widetilde{w}(\cdot, \cdot)$ at the same time and the conclusion of Theorem 3 follows from Theorem 1 . The case $m>k$ can be obtained from the case $m=k$ as follows. Consider $\hat{w}(\cdot, \cdot)$ the solution of the system

$\partial_{t} \hat{w}=\hat{\Sigma}(x) \partial_{x} \hat{w}(t, x)+\hat{C}(x) \hat{w}(t, x)$,

$\hat{w}_{-}(t, 0)=\hat{B} \hat{w}_{+}(t, 0), \quad$ and $\quad \hat{w}_{+}(t, 1)$ are controls.

Here

$\hat{\Sigma}=\operatorname{diag}\left(-\hat{\lambda}_{1}, \ldots,-\hat{\lambda}_{m}, \hat{\lambda}_{m+1}, \ldots \hat{\lambda}_{2 m}\right)$,

with $\hat{\lambda}_{j}=-(1+m-k-j) \varepsilon^{-1}$ for $1 \leq j \leq m-k$ with positive small $\varepsilon, \hat{\lambda}_{j}=\lambda_{j-(m-k)}$ if $m-k+1 \leq j \leq m$, and $\hat{\lambda}_{j+m}=\lambda_{j+k}$ for $1 \leq j \leq m$,

$\hat{C}(x)=\left(\begin{array}{cc}0_{m-k, m-k} & 0_{m-k, n} \\ 0_{n, m-k} & C(x)\end{array}\right)$,

and

$\hat{B}=\left(\begin{array}{cc}I_{m-k} & 0_{m-k, m} \\ 0_{m-k, m} & B\end{array}\right)$,

where $I_{\ell}$ denotes the identity matrix of size $\ell \times \ell$ for $\ell \geq 1$. Recall that $0_{i, j}$ denotes the zero matrix of size $i \times j$ for $i, j, \ell \geq 1$. Then the exact controllability of $w$ at the time $T$ can be derived from the exact controllability of $\hat{w}$ at the same time. One then can deduce the conclusion of Theorem 3 from the case $m=k$ using Theorem 1 by noting that the optimal time for the system of $\hat{w}$ converges to the optimal time for the system of $w$ as $\varepsilon \rightarrow 0_{+}$.

\section{CRediT authorship contribution statement}

Jean-Michel Coron: Conceptualization, Methodology, Writing - review \& editing. Hoai-Minh Nguyen: Conceptualization, Methodology, Writing - review \& editing.

\section{Declaration of competing interest}

The authors declare that they have no known competing financial interests or personal relationships that could have appeared to influence the work reported in this paper.

\section{Acknowledgment}

We thank Guillaume Olive for useful comments on this manuscript.

\section{References}

[1] Georges Bastin, Jean-Michel Coron, Stability and Boundary Stabilization of 1-D Hyperbolic Systems, in: Progress in Nonlinear Differential Equations and their Applications, vol. 88, Birkhäuser/Springer, [Cham], 2016, p. xiv+307, Subseries in Control.

[2] Jean-Michel Coron, Hoai-Minh Nguyen, Optimal time for the controllability of linear hyperbolic systems in one-dimensional space, SIAM J. Control Optim. 57 (2) (2019) 1127-1156, 3932617.

[3] Alberto Bressan, Hyperbolic Systems of Conservation Laws, in: Oxford Lecture Series in Mathematics and its Applications, vol. 20, Oxford University Press, Oxford, 2000, p. xii+250, The one-dimensional Cauchy problem.

[4] David L. Russell, Controllability and stabilizability theory for linear partial differential equations: Recent progress and open questions, SIAM Rev. 20 (4) (1978) 639-739, 508380.
[5] Jean Auriol, Florent Di Meglio, Minimum time control of heterodirectional linear coupled hyperbolic PDEs, Automatica J. IFAC 71 (2016) 300-307, 3521981.

[6] Jean-Michel Coron, Long $\mathrm{Hu}$, Guillaume Olive, Finite-time boundary stabilization of general linear hyperbolic balance laws via Fredholm backstepping transformation, Automatica J. IFAC 84 (2017) 95-100, 3689872 .

[7] Jean-Michel Coron, Rafael Vazquez, Miroslav Krstic, Georges Bastin, Local exponential $\mathrm{H}^{2}$ stabilization of a $2 \times 2$ quasilinear hyperbolic system using backstepping, SIAM J. Control Optim. 51 (3) (2013) 2005-2035, 3049647.

[8] Florent Di Meglio, Rafael Vazquez, Miroslav Krstic, Stabilization of a system of $n+1$ coupled first-order hyperbolic linear PDEs with a single boundary input, IEEE Trans. Automat. Control 58 (12) (2013) 3097-3111, 3152271.

[9] Long $\mathrm{Hu}$, Guillaume Olive, Minimal time for the exact controllability of one-dimensional first-order linear hyperbolic systems by one-sided boundary controls, Preprint, hal-01982662.

[10] Long Hu, Florent Di Meglio, Rafael Vazquez, Miroslav Krstic, Control of homodirectional and general heterodirectional linear coupled hyperbolic PDEs, IEEE Trans. Automat. Control 61 (11) (2016) 3301-3314, 3571452.

[11] Miroslav Krstic, Andrey Smyshlyaev, Boundary Control of PDES, in: Advances in Design and Control, vol. 16, Society for Industrial and Applied Mathematics (SIAM), Philadelphia, PA, 2008, p. $\mathrm{x}+192$, A course on backstepping designs.

[12] Miroslav Krstic, Bao-Zhu Guo, Andras Balogh, Andrey Smyshlyaev, Outputfeedback stabilization of an unstable wave equation, Automatica J. IFAC 44 (1) (2008) 63-74, 2530469.

[13] Andrey Smyshlyaev, Miroslav Krstic, Boundary control of an anti-stable wave equation with anti-damping on the uncontrolled boundary, Systems Control Lett. 58 (8) (2009) 617-623, 2542119.

[14] Andrey Smyshlyaev, Eduardo Cerpa, Miroslav Krstic, Boundary stabilization of a 1-D wave equation with in-domain antidamping, SIAM J. Control Optim. 48 (6) (2010) 4014-4031, 2645471.

[15] Andrey Smyshlyaev, Miroslav Krstic, Closed-form boundary state feedbacks for a class of 1-D partial integro-differential equations, IEEE Trans. Automat. Control 49 (12) (2004) 2185-2202, 2106749.

[16] Andrey Smyshlyaev, Miroslav Krstic, On control design for PDEs with space-dependent diffusivity or time-dependent reactivity, Automatica J. IFAC 41 (9) (2005) 1601-1608, 2161123.

[17] Rafael Vazquez, Miroslav Krstic, Control of 1-D parabolic PDEs with Volterra nonlinearities. I. Design, Automatica J. IFAC 44 (11) (2008) 2778-2790, 2527199.

[18] Jean-Michel Coron, Hoai-Minh Nguyen, Null controllability and finite time stabilization for the heat equations with variable coefficients in space in one dimension via backstepping approach, Arch. Ration. Mech. Anal. 225 (3) (2017) 993-1023, 3667281.

[19] Eduardo Cerpa, Jean-Michel Coron, Rapid stabilization for a Kortewegde Vries equation from the left Dirichlet boundary condition, IEEE Trans. Automat. Control 58 (7) (2013) 1688-1695, 3072853.

[20] Jean-Michel Coron, Qi Lü, Local rapid stabilization for a Korteweg-de Vries equation with a Neumann boundary control on the right, J. Math. Pures Appl. (9) 102 (6) (2014) 1080-1120, 3277436.

[21] Jean-Michel Coron, Ludovick Gagnon, Morgan Morancey, Rapid stabilization of a linearized bilinear 1-D Schrödinger equation, J. Math. Pures Appl. (9) 115 (2018) 24-73, 3808341.

[22] Christophe Zhang, Finite-time internal stabilization of a linear 1-D transport equation, Preprint, hal-01980349, 2019.

[23] Jean-Michel Coron, Control and Nonlinearity, in: Mathematical Surveys and Monographs, vol. 136, American Mathematical Society, Providence, RI, 2007, p. xiv+426, 2302744.

[24] Jeffrey Rauch, Michael Taylor, Exponential decay of solutions to hyperbolic equations in bounded domains, Indiana Univ. Math. J. 24 (1974) 79-86, 361461.

[25] Claude Bardos, Gilles Lebeau, Jeffrey Rauch, Sharp sufficient conditions for the observation, control, and stabilization of waves from the boundary, SIAM J. Control Optim. 30 (5) (1992) 1024-1065, 1178650.

[26] Lionel Rosier, Exact boundary controllability for the Korteweg-de Vries equation on a bounded domain, ESAIM Control Optim. Calc. Var. 2 (1997) 33-55, 1440078.

[27] Michel Duprez, Guillaume Olive, Compact perturbations of controlled systems, Math. Control Relat. Fields 8 (2) (2018) 397-410, 3810881.

[28] Norbert Weck, A remark on controllability for symmetric hyperbolic systems in one space dimension, SIAM J. Control Optim. 20 (1) (1982) $1-8,642174$.

[29] Tatsien Li, Controllability and Observability for Quasilinear Hyperbolic Systems, in: AIMS Series on Applied Mathematics, vol. 3, American Institute of Mathematical Sciences (AIMS), Springfield, MO; Higher Education Press, Beijing, 2010, p. $\mathrm{x}+222$, 2655971 .

[30] Ta-tsien Li, Bopeng Rao, Local exact boundary controllability for a class of quasilinear hyperbolic systems, 23, (2) 2002, pp. 209-218, Dedicated to the memory of Jacques-Louis Lions, 